\title{
Pulsated Thermal Response Test experiments and modelling for ground thermal property estimation
}

Marco Fossa

\begin{abstract}
The Thermal Response Test (TRT) is a well known experimental technique for estimating both the ground thermal conductivity and the effective borehole heat exchangers (BHE) resistance in ground coupled heat pump (GCHP) applications. The usual experimental approach for the TRT measurements is to inject (and even extract) a constant heat rate in the ground while the carrier fluid is circulated inside a reference heat exchanger. In this paper the TRT approach is applied with reference to non constant heat rate condition during a several day measuring session at the SEB building site of the University of Genova, Italy. A constant heat injection has been operated for the first 100 hours of the experiment and then a series of 8 hour square pulses (on/ off mode) have applied for about 11 days. The ground and BHE thermal properties have been here estimated according to different algorithms developed either at the University of Genova and Polytech Montreal, where either the ILS and FLS (Infinite and Finite Line Source) theories or a Resistance/Capacitance approach are implemented to reconstruct the measured temperature evolution from parameter estimation.
\end{abstract}

\section{INTRODUCTION}

The Thermal Response Test is an efficient measurement technique first proposed by P. Mogensen (1983) at the beginning of the $80^{\prime}$ for inferring the ground thermal properties in ground source heat pump (GCHP) applications when borehole heat exchangers (BHE) are employed. As it is well known, during the test, heat carrier fluid temperature is measured at a pilot BHE and the experimental signal is compared to a predicted one when a given (constant) heat transfer rate is provided at the surface. This experimental technique is based on the assumption that the fluid resulting temperature depends on the undisturbed ground one and on a couple of thermal resistances, the (constant) BHE effective resistance and the (transient) ground one, as described by the Infinite Line Source (ILS, Carlaw and Jager, 1947, Ingersoll et al., 1954) model. After Mogensen, early contributions to the TRT technique are due to Gehlin in Sweden (Gehlin 1996), Austin (1998) in the US and Pahud and Matthey (2001) in Switzerland, at the end of the '90. After such experiences the method rapidly developed across the world until its most detailed versions, where multiple vertical measurements are carried out along the BHE for assessing the local ground properties (Distributed TRT at KTH Stockholm, e.g. Acuna et al. 2009).

When the TRT data are processed, a two resistance model is applied where the borehole resistance is conceived to be time invariant and the ground one is set to change in time as described by a suitable Temperature Response Factor (TRF) that from a mathematical point of view is able to describe heat conduction mechanism and provide the borehole periphery temperature evolution as a function of a proper Fourier number. The most popular TRF for TRT analyses is

Marco Fossa (marco.fossa@unige.it) is professor of Solar and Geothermal energy at the University of Genova, Italy. Davide Rolando is post-doctoral researcher at KTH Stockholm, Sweden and Philippe Pasquier is professor of geological engineering at Polytechnique Montreal, Canada 
the ILS solution, but more sophisticated solutions can be chosen, including the Finite Line-Source model (FLS) (Eskilson 1987, Zeng, et al. 2002, Lamarche and Beauchamp 2007), the Moving Infinite Line-Source model (MILS) (Sutton et al. 2003), the Moving Finite Line-Source (MFLS) by Molina-Giraldo et al. (2011) or thermal resistance and capacity (TRC) model such as the one of Pasquier and Marcotte $(2012,2014)$.

The usual assumption related to the TRT experimental data reduction is related to the thermal conduction mechanism and to the absent (or negligible) effects due to the underground water advection. This assumption is usually not fully satisfied, since fractured rocks are often present and some groundwater displacement can be present and enhance the heat transfer from the borehole to the surrounding ground medium, as discussed for example by Gehlin \& Hellstrom (2003) and Spitler \& Gehlin (2015). If groundwater circulation is present the thermal conduction is hence reinforced by convection: in such cases a pure conduction modelling, in terms of moving line sources, demonstrated to be still efficient for describing the ground response to thermal pulses even under groundwater advection. In this sense the works by Sutton et al. (2003) on the MILS and that by Molina-Giraldo et al. (2011) on the MFLS clearly show how a conduction scheme can be efficiently applied for describing the BHE behaviour when groundwater is present.

This paper deals with a particular experimental condition where the heat rate at the heat carrier fluid is deliberately varied according to a series of on/off heat pulses able to resemble a real GCHP operating conditions. A dedicated equipment has been developed to this aim at the University of Genova which is able to very efficiently control the heat and flow rates either in constant or pulsated mode. The measurements carried out in particular refer to a constant heat injection for the first 100 hours of the experiment and to a series of 8 hour square pulses (on/off mode) that have been applied for the following 11 days.

Such Pulsated Thermal Response Test (PTRT) has been analysed with several interpretation models by two different inverse solution algorithms developed at the home universities of the Italian and Canadian authors of the present paper in order to estimate by parameter optimization the ground thermal properties and borehole characteristics, and compare their algorithms. The Italian approach is based on the temporal superposition of the ILS solution and to a cross check with respect to the MILS conditions (Fossa et. al, 2016). Alternatively, the Canadian approach used a convolution is the spectral domain (Marcotte and Pasquier 2008) to account for the heating power variation of the PTRT with the program TRT-SInterp (Pasquier 2015) to automatically calibrate the PTRT with the FLS model of Claesson and Javed (2011) and the TRC model of Pasquier and Marcotte (2012, 2014).

The measurements have been carried out in Italy, and the peculiar geological conditions with non negligible aquifer activity allows the use of either the ILS, MFLS, FLS and TRC models for measured data reduction. Finally, the experimental results and optimum search are presented in order to demonstrate the reliability and accuracy of the experimental apparatus and the parameters that can be estimated according the present modelling approaches.

\section{EXPERIMENTAL APPARATUS AND MEASUREMENTS}

In this paper, the TRT machine developed at Dime, University of Genova (Unige), has been employed for a pulsated TRT performed in Italy at Unige Savona Campus (44¹8'04.6"N 8²7'02.9"E, Smart Energy Building SEB). The machine (Figure 2a) was developed in partnership with the Italian company Erde srl and it is equipped with a feedback control to manage either the flow rate or to the heat rate to the fluid. The heater section is constituted by three $3 \mathrm{~kW}$ electrical resistors controlled through a 3 phase angle power controller (TRIAC), with a control signal in the 0 to $10 \mathrm{~V}$ range (Figure $2 \mathrm{~b}$ ). Analogue signals are provided by a D/A converter that communicates with Linux based microcomputer running a dedicated code. Control works according to a double PI (Proportional and Integral) algorithm and the PI parameters have been tuned following a Ziegler-Nichols [17] approach in dedicated laboratory tests. Temperature measurements are performed through PT1000 RTDs, calibrated to assure $0.1^{\circ} \mathrm{C}$ uncertainty. Mass flow rate and electrical power are available within $2 \%$ accuracy on reading thanks to proper instrumentation calibration performed at Dime. 

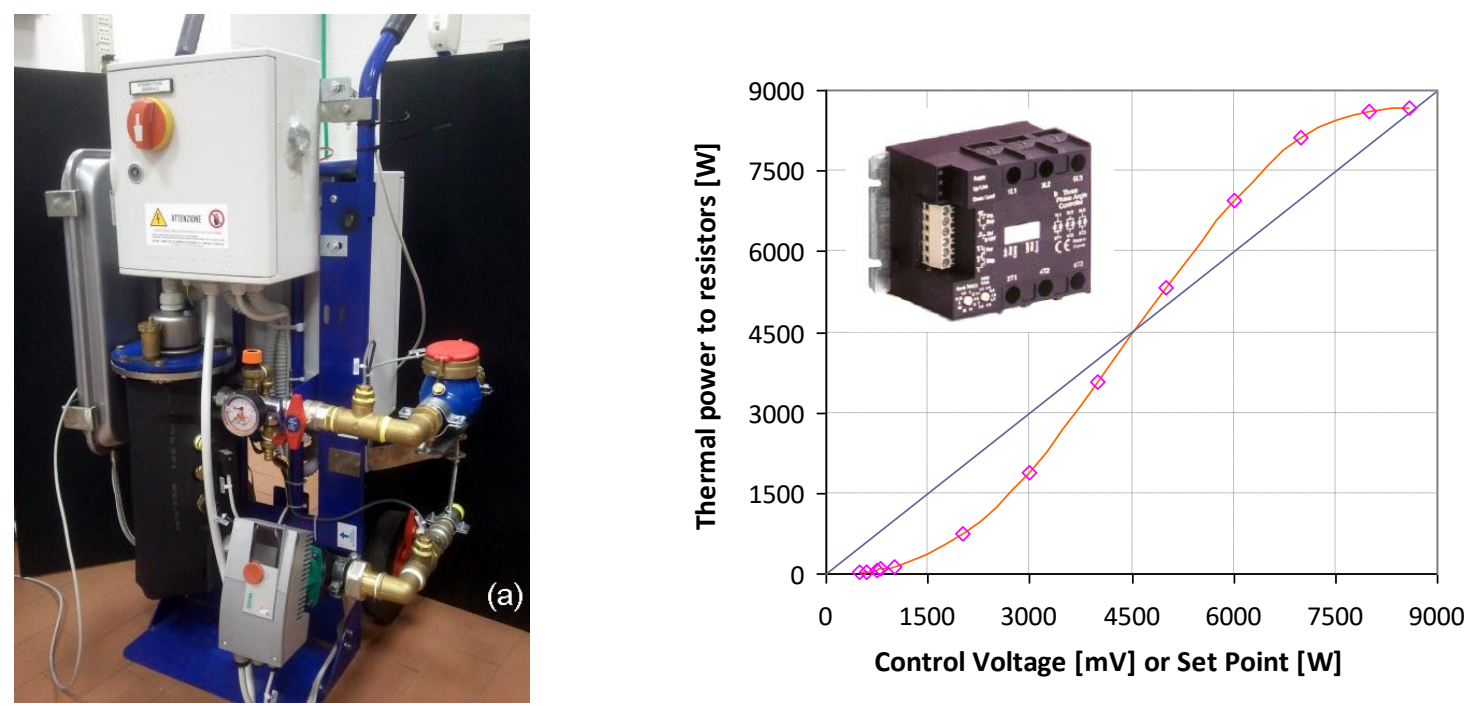

Figure 1 TRT machine developed at the University of Genova (a) and TRIAC power response vs control signal (b).

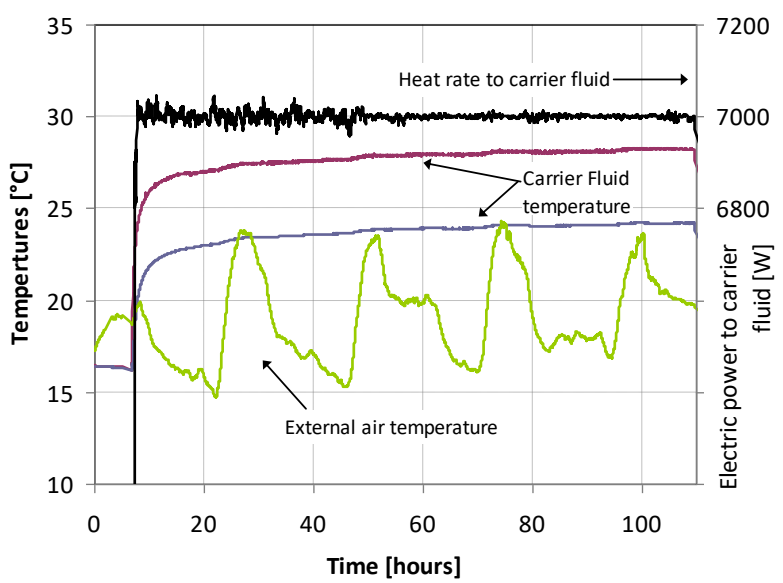

(a)

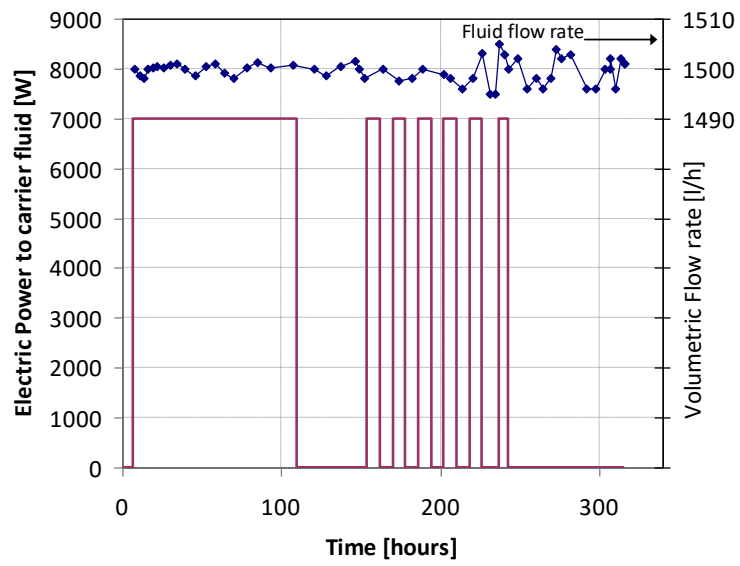

(b)

Figure 2 Temperature profiles during the first 100 hours of the pulsated TRT experiment (a), heat pulse sequence and carrier fluid flow rate vs time (b).

The measurements refer to an experimental campaign dated early November 2015. The pilot heat exchanger was $150 \mathrm{~m}$ deep grouted BHE, working with a single $40 \mathrm{~mm}$ U pipe. Grout nominal conductivity from manufacturer data was $2.0 \mathrm{~W} / \mathrm{m} \mathrm{K}$. Geological surveys revealed that lithology is constituted by a top layer (20-25m deep) of alluvial material subjected to slow underwater movement and by fractured metamorphic formations. The measuring site is also close $(70 \mathrm{~m})$ to the local creek which flows around the University Campus.

The TRT machine was shaded from the sunlight and surface pipes (about $2 \mathrm{~m}$ ) properly insulated, but unusually hot conditions during the measurements slightly affected the carrier fluid temperatures, as can be noticed from the inspection of the fluid temperature records (Figure 2a). The TRT experiment lasted 12 days. The first 7 hours no heat rate was applied (undisturbed temperature measurements) and the following $100 \mathrm{~h}$ a constant heat rate condition was set. From this instant on a series of heat pulses (heater off/on sequences) have been imposed: $45 \mathrm{~h}$ (heating is off) then a series of 12 on and off cycles ( 8 hours each) and a final recovery period (power off) of about $60 \mathrm{~h}$. In the heating 
periods the heat transfer rate was set at $7000 \mathrm{~W}$ and the control was able to maintain a small $1.5 \%$ RMS variation irrespective of the daily supply voltage oscillations. The volumetric flow rate of water was maintained at 1500 liters $/ \mathrm{h}$, with 4 l/h RMS variation (Figure 2b) all along the test.

\section{MODELLING THE HEAT TRANFER INSIDE THE GROUND MEDIUM}

\section{Interpretation with the ILS model}

In GCHP modeling the Fourier conduction equation is very often the modeling approach according to which the variable ground temperatures are predicted when a vertical BHE is considered. As it is well known, the Fourier equation can be either refer to $1 \mathrm{D}$ or 2D temperature domains and different solutions arise from the related boundary conditions applied. The ILS model is probably the most successful scheme since it provides a simple but effective solution according to which when a constant heat transfer rate (per unit length $\dot{Q}^{\prime}$ ) is applied to an extremely long linear source buried inside an infinite medium the ground temperature in space and time can be calculated according the well known relationship, here presented in terms of dimensionless excess temperature $\Gamma$ :

$$
\Gamma_{I L S}=\frac{T(r, \tau)-T_{g r, \infty}}{\dot{Q}^{\prime} / 2 \pi k_{g r}}=\frac{1}{2} \int_{1 / 4 F o_{r}}^{\infty} \frac{e^{-\beta}}{\beta} d \beta=\frac{1}{2} E_{1}\left(1 / 4 F o_{r}\right)
$$

Here above $\mathrm{T}_{\mathrm{gr}, \infty}$ is the initial and far field ground temperature, $\mathrm{k}_{\mathrm{gr}}$ is the ground thermal conductivity, $\mathrm{For}_{\mathrm{r}}$ is the Fourier number based on the radial distance from the line source, $r$. The Exponential Integral $\mathrm{E}_{1}$ can be accurately approximated by its series expansions, as for example discussed by Fossa (2016). Similar solutions have been proposed for referring to heat sources finite in length, as discussed in the Introduction of this paper, but on the other hand it can be demonstrated (e.g. Fossa 2016) that in the short period, typically for $\mathrm{Fo}_{\mathrm{rb}}<10^{4}$, the ILS solution is accurate enough for TRT analysis purposes and it superposes very well to any FLS solution.

A slightly different problem is related to the presence of moving groundwater in the soil. Sutton et al. (2003) and later Molina Giraldo et al. (2011) demonstrated that the moving line source model, still based on the Fourier equation solution, is accurate enough for describing the heat transfer between a single BHE and the surrounding ground when groundwater circulation is present. Sutton et al. in particular described the MILS model, which allows the ground temperature excess to be calculated as a function of the source specific heat rate $\dot{Q}^{\prime}$, the effective ground conductivity $\mathrm{k}_{\mathrm{gr}, \text { eff }}$ and the effective Peclet number ( $\left.\mathrm{Pe}=\mathrm{w}_{\text {eff }} \mathrm{r}_{\mathrm{b}} / \alpha_{\mathrm{gr}, \mathrm{eff}}\right)$. All these effective properties depend either on the characteristics of the porous media and interstitial water. For the MILS, the resulting dimensionless temperature excess $\Gamma$ is:

$$
\Gamma_{\text {MILS }}=\frac{\left(T\left(r_{b}\right)-T_{g r, \infty}\right)}{\dot{Q}^{\prime} / 2 \pi k_{g r, e f f}}=\frac{\int_{0}^{\pi} \exp \left(\frac{P e}{2} \cos (\vartheta) d \vartheta\right)}{2 \pi} \int_{1 / 4 F o_{r b}}^{\infty} \frac{\exp \left(-\zeta-\frac{P e^{2}}{16 \xi}\right)}{\zeta} f \zeta
$$

Molina Giraldo et al. (2011) demonstrated that the MFLS solution is very well approximated by its MILS counterpart for Fourier numbers corresponding to several months of time and that both solutions are in close agreement with 2D numerical solutions of conduction/convection problems in porous ground media with vertical heat exchangers. 


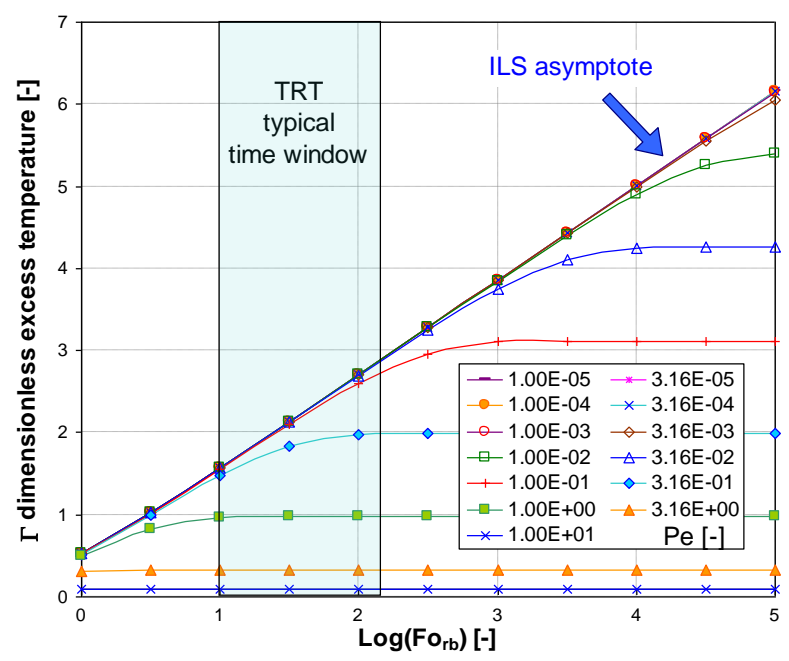

Figure 3. Dimensionless excess temperature $\Gamma$ as a function of Fourier and Peclet numbers for the MILS solution. The TRT band shows the Pe values for ILS applicability to TRT measurements

Recently at Unige the proper and improper integrals of Eq. (2) have been solved and compared to the ILS profile (Eq. 1). Figure 3 shows this comparison in terms of ILS and MILS dimensionless temperature excess. The independent parameters are $\mathrm{Fo}_{\mathrm{eff}}$ and Pe numbers. Figure 3 reveals that for Fourier numbers ranging from 10 to 100 (magnitude orders) the ILS solution is a good approximation of its MILS counterpart, provided that the Peclet number is sufficiently small $(\mathrm{Pe}<0.02)$. Therefore, it has been decided to use the ILS model to interpret the PTRT since both solutions are similar for the duration of the PTRT.

\section{Interpretation with the FLS model}

Although for short TRT duration the ILS and FLS models have the same response, it has been decided to also interpret the PTRT with the FLS model and to observe if integration of the axial effects by the FLS model could lead to different thermal properties for this unusually long TRT. Using the formulation of Claesson and Javed (2011), the dimensionless excess temperature of the FLS model is given by:

$$
\Gamma_{F L S}=\frac{1}{2} \frac{\int_{1}^{\infty}}{\sqrt{4 r_{b}^{2} F o_{r}}} e^{-r_{b}^{2} s^{2}} \frac{Y(H s, D s)}{H s^{2}} d s
$$

with

$$
Y(H s, D s)=2 \operatorname{ierf}(H s)+2 \operatorname{ierf}(H s+2 D s)-\operatorname{ierf}(2 H s+2 D s)-\operatorname{ierf}(2 D s)
$$

and with

$$
\operatorname{ierf}(X)=X \operatorname{erf}(X)-\frac{1}{\sqrt{\pi}}\left(1-e^{-X^{2}}\right)
$$

\section{Interpretation with a TRC model}

To verify if an advanced interpretation model accounting for the capacity of the borehole components could lead to a different set of parameters, the TRC model of Pasquier and Marcotte $(2012,2014)$ was also used to interpret the PTRT. The TRC model integrates the fluid advection within the vertical pipes, the geometry and thermal conductivity and capacity of each component (fluid, pipe, grout, and ground) through a network of interconnected thermal resistances $(R)$ and capacities $(C)$ chosen to ensure a high accuracy of the interpretation model. For a network of $n$ 
nodes, the model allows writing heat conservation at node $j$ by

$$
C_{j} \frac{d T_{j}}{d t}=\sum_{k=1}^{n_{j}} \frac{T_{k}-T_{j}}{R_{k}}
$$

where $n_{j}$ is the number of neighbouring nodes to node $j$. Assembling Eq. 6 for all nodes of the model leads to a stiff first-order differential equations system whose solution is obtained by numerical integration with the Haae wavelet method (Nguyen and Pasquier 2015).

\section{PARAMETER ESTIMATION FROM TRT MEASUREMENTS}

The interpretation of a TRT can be accomplished by formulating the parameter identification task as an inverse problem. The interpretation strategy then consists to minimize an objective function quantifying the misfit between the temperatures simulated by an interpretation model and the temperatures actually measured on the field. This interpretation method is now used frequently (Wagner and Clauser 2005; Marcotte and Pasquier 2008) and permits using interpretation models that incorporate directly significant variations of the heating power. The use of a more accurate interpretation model often leads to a more robust parameter identification.

For the ILS and FLS models, the evolution of the mean fluid temperature under the constraint of a variation of the heating power signal, as in a PTRT, can be formulated as a convolution product by

$$
T_{f, a v e}\left(\tau_{N}\right)=T_{g r, \infty}+\dot{Q}_{N}^{\prime} R_{b h e}+\frac{1}{2 \pi k_{g r}} \sum_{i=1}^{N}\left(\dot{Q}_{i}^{\prime}-\dot{Q}_{i-1}^{\prime}\right) \Gamma\left(F o\left(\tau_{N}-\tau_{i-1}\right)\right)
$$

where $R_{b b e}$ is the equivalent borehole resistance and the index $i$ refers to the time step index. From Eq.7, the optimization procedure consists to find the $\mathrm{R}_{\mathrm{bhe}}$ and $\mathrm{k}_{\mathrm{gr}}$ couple that minimizes the discrepancy between the experimental and modelled temperatures.

The procedure used with the ILS model is based on trial and error. Starting with initial guesses of $\mathrm{k}_{\mathrm{gr}}$ and $\mathrm{R}_{\mathrm{bhe}}$, the simulated fluid temperatures are compared to the average fluid temperature $\mathrm{T}_{f, a v e}$ and the parameters are changed until an optimum solution is found. To interpret the PTRT with the FLS and TRC models, the program TRT-SInterp (Pasquier 2015) was used. The latter is dedicated to stochastic and deterministic interpretation of TRTs by optimization. Since its initial publication, the program has undergone constant improvements and now includes a graphical user interface to ease its use in the industry.

\section{RESULTS AND DISCUSSION}

The parameter recognition procedures adopted in this paper has been used with the ILS, FLS and TRC models to interpret the PTRT. The parameters found at the optimum along with the calibration statistics are summarized in Table 1. In addition, Figure 4 compares the experimental temperatures and the temperatures simulated by the various interpretation models used in this work with the parameters found at the optimum. The thermal conductivity values range from 6.17 to $6.25 \mathrm{~W} / \mathrm{mK}$. Although the values obtained are quite high, these results are in agreement with a

Table 1: Thermal parameters obtained after interpretation of the PTRT

\begin{tabular}{cccc}
\hline $\begin{array}{c}\text { Interpretation } \\
\text { model }\end{array}$ & $\begin{array}{c}\text { Thermal } \\
\text { conductivity } \\
\text { (W/mK) }\end{array}$ & $\begin{array}{c}\text { Borehole } \\
\text { Resistance } \\
\text { (mK/W) }\end{array}$ & $\begin{array}{c}\text { Mean error on predicted } \\
\text { temperature } \\
\text { (K) }\end{array}$ \\
\hline \hline ILS & 6.25 & 0.122 & 0.10 \\
FLS & 6.17 & 0.131 & 0.11 \\
TRC & 6.24 & 0.128 & 0.16 \\
\hline
\end{tabular}




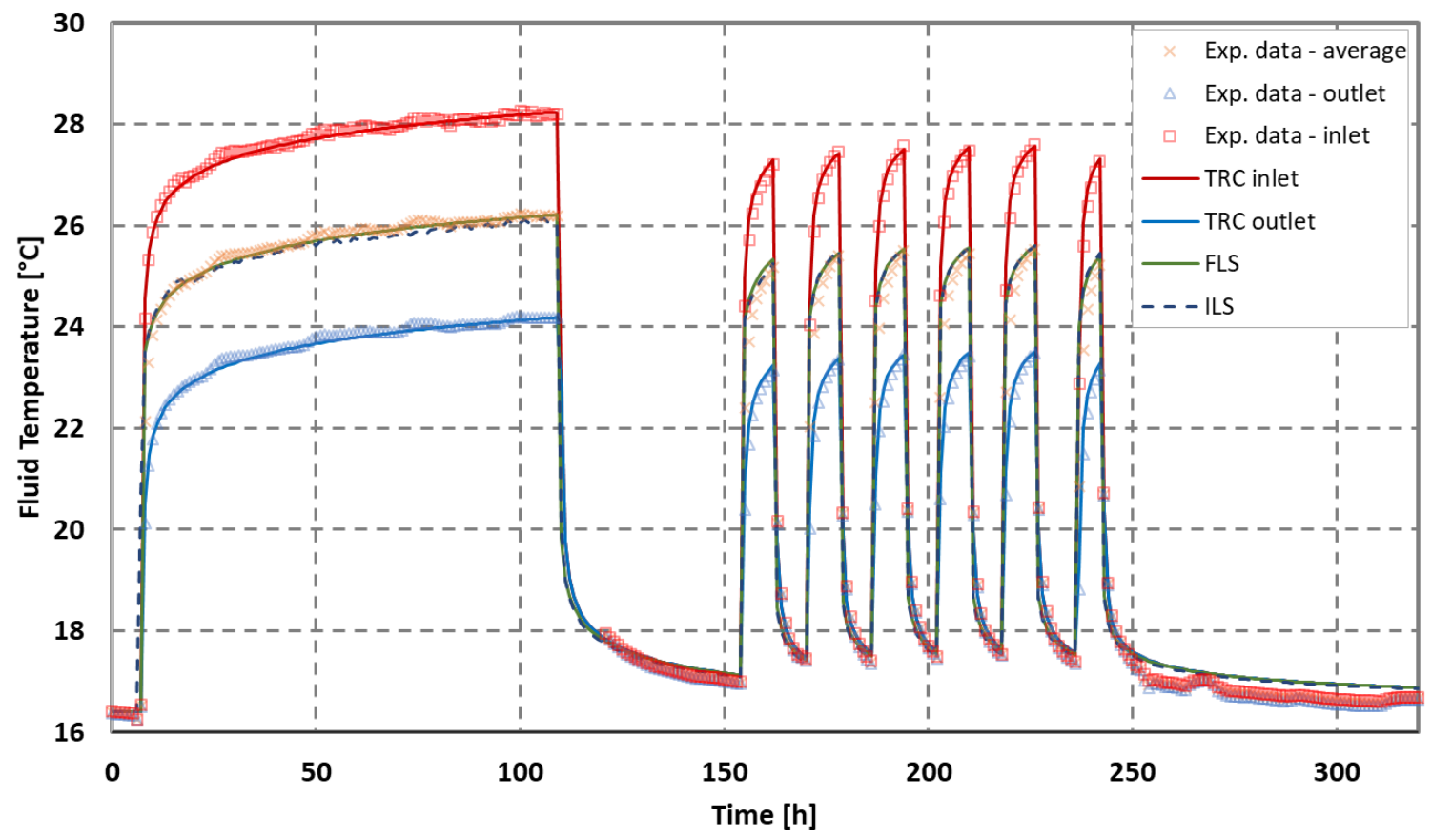

Figure 4. Comparion of the experimental temperatures with the ILS, FLS and TRC models used to interpret the PTRT.

value obtained independently on the same BHE in August 2015 by a private company having its own standard TRT unit and applying its own interpretation procedures. The thermal conductivity values have hence been ascribed to the particular local lithology, where the circulation of groundwater seems plausible. The reduced slope of alluvial plane $(2.5 \%)$ and the estimated permeability of the top layer around $10^{-5} \mathrm{~cm} / \mathrm{s}$ are finally compatible with Pe numbers related to the validity of ILS model to the present enhanced TRT analysis. However, at this point it is unclear if the high thermal conductivity observed is caused by a significant groundwater flow or is simply the expression of a highly conductive geological material.

It is reassuring to see similar thermal parameters, regardless of the interpretation model used. Indeed, the parameters found at the optimum differ than less than $1.3 \%$ for $k_{g r}$ and $6.9 \%$ for $R_{b b e}$. For the interpretation of this specific PRTR, the variations observed are attributed mainly to the different convergence parameters used to control the optimization algorithms. It should be noted that this PTRT induced a narrow valley for the objective function in the parameter space that was sometimes a challenge for the optimization algorithms.

It should be noted that from the author's experience, obtaining borehole equivalent resistance with the ILS/FLS models that is so close to the one obtained with the TRC model is unusual. When interpreting a conventional TRT, the thermal conductivity obtained are quite similar but the borehole resistance provided by the TRC model is usually better identified. These results might indicate that performing a long PTRT can filter out the impact of the interpretation model on the interpretation outcome and improve the identification of $R_{b b e}$.

The thermal parameters found reproduce also quite well the experimental temperatures measured during the PTRT as seen in Figure 4. Indeed, the output of all three interpretation models adjust quite well the experimental temperatures. Note how the TRC model was slightly better at adjusting the peaks corresponding the each pulse, but this did not resulted in a significantly better adjustment from an overall statistic error. All three models are able to reproduce correctly also the long recovery phases, even if a closer inspection of numerical values would reveal discrepancies within tens of degree. A thermal contamination of the measurements or a significant groundwater flow could explain the inability of the models to fit the long recovery phases. However, for now this remains an open 
problem.

\title{
CONCLUSIONS
}

In this paper the measurements from a pulsated Thermal Response Test have been presented and discussed. In this particular experiment the heat rate at the carried fluid was deliberately varied according to a series of on/off heat pulses able to resemble a real GCHP operating conditions. A dedicated equipment has been developed to this aim at the University of Genova which is able to very efficiently control the heat and flow rates either in constant or pulsated mode. Such a Pulsated Thermal Response Test has been processed by 3 different inverse solution algorithms developed at the home universities of the Italian and Canadian authors of the present paper. The methods include the application of the temporal superposition techniques, the use of the ILS and FLS solutions for the semi-infinite ground medium and even a resistance/capacitance method (the TRC algorithm) able to take into consideration whole the inner borehole thermal network. Very similar thermal parameters have been estimated by the three algorithms: at convergence the results differ less than $1.3 \%$ for $k_{g r}$ and $6.9 \%$ for $R_{b b e}$. Based on author's experience, a similar agreement on $R_{b b e}$. estimations between TRC and ILS/FLS models is pretty unusual. These results might indicate that performing a long PTRT can filter out the impact of the interpretation model on the interpretation outcome and improve the identification of borehole thermal resistance.

\section{ACKNOWLEDGMENTS}

Dime Dept. at University of Genova is greatful to MAVOCO Gmbh for the support provided in measurement data transfer via dedicated machine-to-machine protocol.

\section{NOMENCLATURE}

$$
\begin{aligned}
\mathrm{E}_{1} & =\text { Exponential Integral solution }(-) \\
\Gamma & =\text { temperature response factor }(-) \\
\mathrm{F}_{\mathrm{r}} & =\text { radius based Fourier number }(-) \\
\mathrm{Pe} & =\text { Peclet number }(-) \\
\dot{Q}^{\prime} & =\text { specific heat rate }(\mathrm{W} / \mathrm{m}) \\
\alpha & =\text { ground thermal diffusivity }\left(\mathrm{m}^{2} / \mathrm{s}\right) \\
\mathrm{C} & =\text { thermal capacity }\left(\mathrm{J} / \mathrm{m}^{3} \mathrm{~K}\right) \\
\mathrm{k}_{\mathrm{gr}} & =\text { ground thermal conductivity }(\mathrm{W} / \mathrm{mK})
\end{aligned}
$$

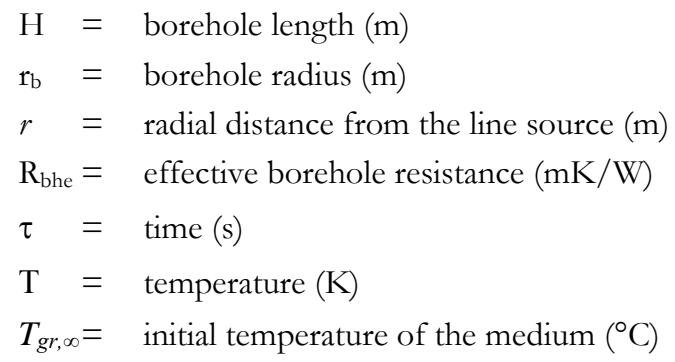

\section{SUBSCRIPTS}

\author{
FLS $=$ Finite Line Source \\ ILS $=$ Infinite Line Source \\ MILS $=$ Moving Infinite Line Source \\ eff $=$ effective, as weighted average between soil and water properties
}

\section{REFERENCES}

Acuna J., P. Mogensen and B. Palm, 2009. Distributed thermal response test on a U-pipe borehole heat exchanger. In: 11th Int. Conf. on Energy Storage EFFSTOCK, Stockholm.

Austin, W A. 1998. Development of an in situ system for measuring ground thermal properties, PhD Thesis, Oklahoma State University. Carslaw, H.S and J.C. Jaeger, 1947. Conduction of heat in solids. Claremore Press Oxford UK. 
Claesson J. and S. Javed, 2011. An Analytical Method to Calculate Borehole Fluid Temperatures for Time-scales from Minutes to Decades. ASHRAE Transactions 117:279-288.

Eskilson P., 1987. Thermal analysis of heat extraction boreholes. Ph.D. Thesis, Lund University of Technology, Sweden.

Fossa M., A. Priarone, D. Rolando and F. Berti, 2016. Borefield Design and Enhanced Thermal Response Test for the Energy Building of the University of Genova, UIT Heat Transfer Conference, Ferrara, Italy.

Fossa, M., 2016. Correct Design of Vertical BHE Systems Through the Improvement of the Ashrae Method, Science and Technology for the Built Environment, 1-10

Gehlin, S., 1996. Thermal response test method development and evaluation Licentiate Thesis, Lulea University of Technology.

Gehlin, S.E.A. and G. Hellström, 2003. Influence on Thermal Response Test by Groundwater Flow in Vertical Fractures in Hard Rock. Renewable Energy 28: 2221-2238.

Hellström G., 1991. Ground Heat Storage. Thermal Analysis of Duct Storage Systems. Part I Theory. University of Lund, Sweden.

Ingersoll, L.R., O.J. Zobel and A.C. Ingersoll, 1954. Heat conduction with engineering, geological, and other applications. McGrawHill New York.

Lamarche, L. and B. Beauchamp, 2007. A new contribution to the finite line-source model for geothermal boreholes, Energy Build. 39: 188-198.

Marcotte D. and P. Pasquier, 2008. On the estimation of thermal resistance in borehole thermal conductivity test. Renewable Energy 33(11):2407-15.

Mogensen, P. 1983. Fluid to duct wall heat transfer in duct system heat storages Document-Swedish Council for Building Research 16: 652657.

Molina-Giraldo N, P. Blum, K. Zhu, P. Bayer and Z. Fang, 2011. A moving finite line source model to simulate borehole beat exchangers with groundwater advection. International Journal of Thermal Sciences 50(12):2506-13.

Molina-Giraldo, N., P. Blum, K Zhu, P Bayer and Z Fang. 2011. A moving finite line source model to simulate borehole beat exchangers with groundwater advection. Int. J. Therm. Sci 50: 2506-2513.

Nguyen A and P. Pasquier, 2015. An adaptive segmentation Haar wavelet method for solving thermal resistance and capacity models of ground heat exchangers. Applied Thermal Engineering 89:70-9.

Pahud, D and B. Matthey, 2001. Comparison of the thermal performance of double U-pipe borehole heat exchangers measured in situ. Energy and buildings 33: 503-507.

Pasquier P, D. Marcotte, 2014. Joint use of quasi-3D response model and spectral method to simulate borehole beat exchanger. Geothermics. 51:281-99.

Pasquier P. and Marcotte D., 2012. Short-term simulation of ground heat exchanger with an improved TRCM. Renewable Energy 46:92-9.

Pasquier P., 2015. Stochastic interpretation of thermal response test with TRT-SInterp. Computers \& Geosciences 75:73-87.

Spitler, J. D. and S. E. A. Gehlin, 2015. Thermal response testing for ground source heat pump systems-An historical review. Renewable and Sustainable Energy Reviews 50(0): 1125-1137.

Sutton, M.G., D.W. Nutter and R.J. Couvillion. 2003. A ground resistance for vertical bore heat exchangers with groundwater flow. J. Energy Resources. Technology 125(3): 183-189.

Wagner R, C. Clauser, 2005. Evaluating thermal response tests using parameter estimation for thermal conductivity and thermal capacity. Journal of Geophysics and Engineering 2(4):349-56.

Zeng H.Y., N.R. Diao and Z.H. Fang, 2002. A Finite line-source model for boreholes in geothermal heat exchangers, Heat Transfer-Asian Research, 31: 558-567.

Ziegler, J.G. and N.B. Nichols, 1942. Optimum settings for automatic controllers Transaction ASME 64 (11). 